Cahiers de recherches médiévales

\title{
La Passion de vengeance dans les Nouvelles Récréations et Joyeux Devis de Bonaventure Des Périers
}

\section{Bénédicte Boudou}

\section{(2) OpenEdition Journals}

Édition électronique

URL : https://journals.openedition.org/crm/11537

DOI : $10.4000 / \mathrm{crm} .11537$

ISSN : 1955-2424

Éditeur

Honoré Champion

Édition imprimée

Date de publication : 15 juin 2009

Pagination : $313-328$

ISSN : $1272-9752$

Référence électronique

Bénédicte Boudou, «La Passion de vengeance dans les Nouvelles Récréations et Joyeux Devis de Bonaventure Des Périers », Cahiers de recherches médiévales [En ligne], 17 | 2009, mis en ligne le 15 juin 2012, consulté le 15 décembre 2022. URL : http://journals.openedition.org/crm/11537 ; DOI : https:// doi.org/10.4000/crm. 11537 


\title{
RM
}

\author{
La Passion de vengeance \\ dans les Nouvelles Récréations et Joyeux Devis \\ de Bonaventure Des Périers
}

\begin{abstract}
In the preamble to his Nouvelles Récréations et Joyeux Devis, Bonaventure des Périers expresses the desire to entertain in order to keep sorrows at bay. Thus passions, which count among the sorrows, receive very little attention in Des Périers' work. However, in so far as as his short stories are centred on social relations, approximately fifteen of them involve cases of revenge. Sometimes this vengeance is contained in a witty reply or motto, sometimes in a beffa. The narrator prefers harmless verbal revenge, while underlining the brutality of certain vengeances that apply the law of talion, and that are incommensurate with the fault or offence. For des Périers, Patience seems the best response to offence: is it not better to avoid anger and scandal? Des Périers thus invites his reader to adopt an attitude of «invincible constancy».
\end{abstract}

Résumé: Dès le préambule des Nouvelles Récréations et Joyeux Devis, Bonaventure des Périers affiche une volonté récréative qui cherche à tenir les chagrins à distance. Les passions font partie de ces chagrins, et Des Périers ne leur fait guère de place. Pourtant, dans la mesure où ses nouvelles sont centrées sur des rapports sociaux, une quinzaine d'entre elles présentent des cas de vengeance. Tantôt cette vengeance tient en un bon mot ou motto, tantôt elle donne lieu à une beffa. Le conteur privilégie les vengeances verbales qui ne portent pas dommage à autrui, tandis qu'il souligne la brutalité de certaines vengeances qui, sans mesure avec la faute ou l'offense, appliquent la loi du talion. La patience lui semble la meilleure attitude devant l'offense: ne vaut-il pas mieux éviter l'ire et le scandale? Des Périers invite ainsi son lecteur à une «invincible constance».

Les Nouvelles Récréations restent un livre assez mystérieux, comme le rappelle Krystyna Kasprzyk qui les a éditées en 1980 à la Société des Textes Français Modernes ${ }^{1}$. Publié en 1558, quinze ans après la mort énigmatique de Bonaventure des Périers, le recueil comporte quatre-vingt-dix récits, dont les plus longs dépassent rarement cinq pages, et qui s'inscrivent dans la droite ligne de la tradition facétieuse du Pogge (auquel Des Périers emprunte un certain nombre de récits) ${ }^{2}$. Dans la préface de ses Facéties, le Pogge rappelait ${ }^{3}$ : «Il est bon en effet et même nécessaire, et du reste conforme aux préceptes des philosophes, d'arracher parfois notre esprit à ses habituelles préoccupations et aux fatigues qui l'accablent

\footnotetext{
${ }^{1}$ Toutes nos références de pages renverront à cette édition des Nouvelles Récréations et Joyeux Devis, que nous appellerons désormais $N R$.

${ }^{2}$ Des Périers / Pogge : 2/217, 9/223, 24/90, 59/203, 68/268, 76/147, 88/250.

${ }^{3}$ Le Pogge se situe dans la ligne du De Sermone de Giovanni Pontano, dont le livre VI définit le genre narratif et comique comme récréation visant à nous relaxer.
}

Cahiers de Recherches Médiévales, 17, 2009 
en l'égayant par quelque joyeux délassement ${ }^{4}$. C'est explicitement dans cette perspective que se situe Des Périers, dont la «premiere nouvelle en forme de preambule» joue le double rôle de pacte de lecture et d'art de vivre. Françoise Charpentier $^{5}$ et Krystyna Kasprzyk rapprochent du prologue de Gargantua ce préambule qui rejette la recherche d'un plus haut sens ${ }^{6}$. S'adressant au lecteur, le narrateur insiste sur la diversité des nouvelles qu'il présente et sur la liberté de la lecture qui n'est pas assujettie à un ordre quelconque puisque la composition du recueil n'en a pas suivi ${ }^{7}$. Ses récits sont dénués de caractère anecdotique ${ }^{8}$. Le préambule qui se fait éloge du rire constitue encore un art de vivre qui donne au recueil son orientation: Bene vivere et lateri. Cette perspective récréative n'est pas seulement un topos de la littérature narrative. Elle s'inscrit dans un contexte politique et religieux assez tourmenté, que le narrateur esquisse dans les premières phrases de son recueil avec une discrétion qui ne doit pas nous abuser ( Je vous gardoys ces joyeux propos à quand la paix seroit faicte...», p. 13). Les Nouvelles Récréations ont probablement été composées entre 1540 et 1544 , date présumée de la mort de Des Périers. À cette époque, le roi a cessé de protéger les évangéliques et a fait poursuivre l'un des éditeurs du Cymbalum mundi, Jean Morin. On ignore si Des Périers est encore au service de Marguerite de Navarre, et ce n'est pas en vain qu'il fait allusion à la dureté des temps.

Le sonnet liminaire nous permet de mieux comprendre le sens que Des Périers donne au mot «resjouyssance». Le narrateur s'y adresse à un public choisi : «Hommes pensifz» (p. 2, v. 1). En faisant suivre le mot « chagrin » du mot « ire» :

Laissez à part vostre chagrin, vostre ire,

le vers 5 approfondit le sens du premier terme : la colère est une des formes les plus fréquentes du chagrin. Le narrateur invite ses lecteurs à se maîtriser comme luimême s'est « contrainct » pour écrire.

Un tel vocabulaire nous renvoie à la philosophie stoïcienne que Des Périers connaissait bien: il est en effet l'auteur probable d'une traduction du traité des Quatre vertus attribué à Sénèque'. Mais à cela s'ajoute une culture qu'il a acquise dans le cercle de Marguerite de Navarre. On se rappelle que Lefèvre d'Étaples a par exemple édité en 1530 l'Éthique à Nicomaque d'Aristote, traité qui, avec ceux de Sénèque et de Cicéron, irrigue la pensée morale du $\mathrm{XVI}^{\mathrm{e}}$ siècle. Le conseil que prodiguent les vers 11 et 12 du sonnet liminaire :

\footnotetext{
${ }^{4}$ Poggio Bracciolini (ou le Pogge), Facéties, «Avis aux envieux de ne pas censurer le ton léger des Facéties », éd. Étienne Wolff, Anatolia, 1994, p. 37.

5 «Une page rabelaisienne de Des Périers : la Première nouvelle en forme de préambule », Revue d'Histoire Littéraire de la France, 1967, 3, p. 601-615.

${ }^{6} N R$, p. 15 : «Il n'y a point de sens allegoricque, mistique, fantastique ». Voir la note 4.

${ }^{7} N R$, p. 15 : «Et ne me venez point demander quelle ordonnance j'ay tenue. Car quel ordre fault il garder quand il est question de rire ? » et «Ouvrez le livre : si ung compte ne vous plaist, hay à l'autre ».

${ }^{8} N R$, p. $15:$ : [...] Ne vous chaille si ce fut Gautier ou si ce fut Garguille. Ne vous souciez point si ce fut à Tours en Berry, ou à Bourges en Touraine».

${ }^{9}$ Lionello Sozzi, Les Contes de Bonaventure Des Périers, Contribution à l'étude de la nouvelle française de la Renaissance, Turin, 1965, Genève, Slatkine rep. 1998, p. 49.
} 
Donnons, donnons quelque lieu à folie,

Que maugré nous ne nous vienne saisir.

semble ainsi venir d'Aristote ${ }^{10}$ relu par Sénèque ${ }^{11}$ : «Nulle âme n'est exempte de folie ». La faculté de se réjouir implique donc d'abord un effort, en même temps qu'une grande souplesse pour accepter le présent: «Prenez le temps comme il vient $\gg^{12}$. Savoir se réjouir exige encore de refuser le chagrin inutile ${ }^{13}$, où se reconnaît le mieux ce qui définit la passion ${ }^{14}$. La «premiere nouvelle en forme de preambule» tourne le dos à l'ataraxie stö̈cienne en acceptant l'existence des passions, même chez Socrate dont le narrateur ne peut accepter «qu'il fust ainsi sans passions $»^{15}$. L'aptitude à se réjouir se substitue aux remèdes ${ }^{16}$ prodigués aux hommes chagrins, et le narrateur oppose la patience à l'expérience douloureuse de la passion. Par là, ce prologue fait écho à l'analyse que Cicéron propose des consolations du chagrin dans les Tusculanes: «Le premier remède, c'est de faire comprendre que le sujet du chagrin n'est pas un mal, ou que c'est un très faible mal; le second, c'est de développer des vues sur la condition humaine, en insistant sur la situation particulière à la personne affligée; le troisième, c'est qu'il est extrêmement déraisonnable et vain d'être accablé d'un chagrin qui ne peut servir à rien ${ }^{17}$.

À la fin du prologue, l'exemple du plaisantin qui brave la mort ${ }^{18}-$ et qui peut incarner l'attitude des cyniques opposée à l'intellectualisme de Socrate - illustre à la fois le pouvoir des passions sur les hommes et la mise à distance qu'il est possible de leur opposer. On retrouve cette distanciation dans le recueil des Nouvelles Récréations, qui s'applique parfois à prendre le contre-pied du Livre du Courtisan, en particulier du quatrième dialogue néo-platonicien sur l'amour, et lui préfère la peinture de travers tels que la coquetterie des femmes ${ }^{19}$, la gourmandise ${ }^{20}$, l'ivrognerie $^{21}$ ou encore l'impatience ${ }^{22}$. Si quelques-unes des nouvelles de Des

\footnotetext{
${ }^{10}$ Problèmes, XXX, 1.

${ }^{11}$ De Tranquillitate animi, XVII, 10 : siue Aristoteli, "nullum magnum ingenium sine mixtura dementia fuit".

${ }^{12} N R$, p. 14.

${ }^{13} N R$, p. $14:$ : Ne vous chagrinez point d'une chose irremediable».

${ }^{14}$ Voir Thomas d'Aquin, Somme théologique, $\mathrm{I}^{\mathrm{a}} \mathrm{II}^{\mathfrak{x}}$, quest. 35, art. 2 : la tristesse est la douleur qui vient d'une connaissance intérieure.

${ }^{15} \mathrm{NR}$, p. 17.

${ }^{16} N R$, p. 14 : «Il fault reprimer son courroux : l'autre, peu parler : l'autre, croire conseil : l'autre, estre sobre : l'autre, faire des amis ».

${ }^{17}$ Cicéron, Tusculanes, III, XXXII, 77, Les Stoïciens, trad. E. Bréhier, éd. P.-M. Schuhl, Gallimard, 1962, p. 325 (Erit igitur in consolationibus prima medicina docere aut nullum malum esse aut admodum parvum, altera et de communi condicione vita et proprie, si quid sit de ipsius qui mareat disputandum, tertia summam esse stultitiam frustra confici marore, cum intellegas nihil posse profici).

${ }^{18} N R$, p. $18-19$.

${ }^{19} N R 54$ et 64 .

${ }^{20} N R 57$ et 73

${ }^{21} N R 77$ et 85 , par exemple.

${ }^{22} N R 41$ et 45 .
} 
Périers s'attachent à un seul personnage, la plupart d'entre elles est plutôt centrée sur des relations, en particulier des situations de conflit opposant des voisins ${ }^{23}$, ou des personnages appartenant à des classes sociales différentes. Ces échanges, souvent inégalitaires, font naître des différends qui mettent parfois en jeu la passion de la colère et le désir de vengeance qui l'accompagne. Comme c'était déjà le cas dans le Décaméron de Boccace, la sottise et la ruse qui lui répond constituent des caractéristiques génériques de la nouvelle chez Des Périers. On pourrait donc s'attendre à ce que soit saluée une vengeance habilement menée. Pourtant, le narrateur, loin de considérer toute vengeance comme légitime, laisse entendre des réserves qui ressortissent à l'évangélisme contre la passion vindicative qui cherche à nuire à l'autre, et non à le corriger. La vengeance qui désire le $\mathrm{mal}^{24} \mathrm{n}$ 'est pas traitée de la même façon que celle qui corrige les vices, et malgré la distance que cherche à garder le narrateur, on reconnaîtra certaines prises de parti dans ses récits. Enfin, on s'interrogera sur le rapport qui existe entre la vengeance et la patience déployée dans les épreuves, ou ce que le prologue des Nouvelles Récréations appelle la résistance au chagrin.

\section{Les récits de vengeance: typologie}

Une quinzaine des quatre-vingt-dix récits des Nouvelles Récréations mettent en scène la vengeance. Alors qu'il promet de «joyeux propos », il est à noter que Des Périers ne tire aucunement parti du motif - consacré dans les contes plaisants de la vengeance insignifiante qui ridiculise le vengeur. Présente dans le recueil bourguignon des Cent nouvelles nouvelles ${ }^{25}$ de Philippe de Vigneulles, la vengeance insignifiante consiste à ne pas se venger directement de l'offenseur, mais à s'en prendre à son cheval ou à ses souliers, par exemple. Henri Estienne raille ainsi : «[...] celuy qui se doutant que sa femme ne couchoit pas seule en son absence, vint une fois pour la surprendre : et ayant apperceu devant le lict les souliers de celuy qui estoit pour lors son lieutenant, Voilà (dict-il) de bonnes enseignes: je me contente d'avoir veu cela. Ce qu'ayant dict s'en alla tout bellement, les laissant achever ce qu'ils avoyent commencé. Le lendemain matin vint trouver ses parens et amis, et apres leur avoir conté le faict, Regardez (dict-il) comment la cholere transporte quelquesfois les hommes : car quand j'apperceu devant le lict les souliers de celuy qui estoit couché avec ma femme, il ne s'en falut guere que je ne misse ces souliers en mille pieces ${ }^{26}$. Il est significatif qu'on ne rencontre pas ce type de vengeance chez Bonaventure des Périers, qui préfère donner aux faibles l'occasion de prendre leur revanche contre ceux qui les oppressent. Il choisit de mettre en scène soit l'accomplissement d'une vengeance, soit son évitement, et deux schémas de vengeance se rencontrent dans les Nouvelles Récréations. Cinq récits ${ }^{27}$ évoquent des

\footnotetext{
${ }^{23}$ NR 18, 19, 67.

${ }^{24}$ Voir Thomas d'Aquin, ouvrage cité, $\mathrm{II}^{\mathrm{a}} \mathrm{II}^{\mathfrak{x}}$, quest. 158, art. 1, sol. 3

${ }^{25}$ Voir en particulier $N R$ 17, 43, 69, 70, et le commentaire de Charles H. Livingstone, éd. des Cent Nouvelles nouvelles, Droz, 1972, p. 186.

${ }^{26}$ Henri Estienne, Apologie pour Hérodote, chap. Xv, éd. Bénédicte Boudou, Droz (TLF), 2007, t. I, p. 367.

${ }^{27} N R 10,44,56,80,82$.
} 
cas de vol ou d'offense publique, tandis que plus de dix nouvelles ${ }^{28}$ sont consacrées à des vengeances réagissant à des tromperies ou à des offenses privées, dont quatre ${ }^{29}$ relatives à l'adultère que cherche à punir l'époux trompé. Or la colère est essentiellement une passion sociale, qu'Aristote définit même comme «un désir accompagné de peine, de se venger ostensiblement d'une marque de mépris manifesté à notre égard, ou à l'égard de ce qui dépend de nous, contrairement à la convenance $»^{30}$. La disproportion qu' on observe dans les Nouvelles Récréations entre les vengeances privées et les vengeances publiques, et la prépondérance que Des Périers confère à la vengeance privée sont intéressantes parce qu'est ainsi dénoncé le recours abusif à la passion vindicative, qui est liée au regard d'autrui. À cette première distinction s'ajoute le fait que, dans ces nouvelles, la place accordée à la vengeance comme telle est variable. Ainsi, dix lignes suffisent à Des Périers dans la nouvelle 56, où la victime d'un coupe-bourse se venge immédiatement en coupant l'oreille de celui qui lui a volé ses boutons (p. 212), tandis que la nouvelle 6, qui lui est antithétique puisqu'elle est consacrée au refus de vengeance d'un mari trompé, occupe huit pages (p. 38 à 43).

Si la brièveté de la nouvelle 56 suggère une forme d'incompatibilité entre l'application de la loi du talion et l'invention narrative, les récits de vengeance se répartissent en deux catégories héritées des nouvelles italiennes, les motti et les beffe. Quelques vengeances tiennent en de bons mots et répondent au schéma de la facétie que définissait Castiglione dans le Livre du Courtisan: «Ces bons mots ont beaucoup de grâce: quand quelqu'un reprend dans le propos mordant de son interlocuteur les mêmes termes dans le même sens et les retourne contre lui en le blessant $»^{31}$. C'est ce qui se produit dans la nouvelle 21 , dont le protagoniste, un écolier frais émoulu de l'enseignement du latin classique, est examiné par un prêtre qui substitue toujours à sa langue classique un latin macaronique. L'écolier finit par se venger de ce prêtre en reprenant le latin macaronique du clerc pour l'avertir d'un incendie qu'il a allumé chez lui : «Prestole, mitis habet gaudium in suavi : quod si abundantia non est, tu amittis tuum requies $»^{32}$. La nouvelle 31 évoque l'échange de madame «La Fourriere» avec un gentilhomme qui l'a laissée insatisfaite. À l'attaque du gentilhomme «Vous me logeastes l'aultre nuict bien au large», elle rétorque «Il est vray, monsieur, [...] Mais je ne pensois pas que vous eussiez si petit train $»^{33}$. La nouvelle, qui a pris sa source dans la facétie, constitue un terrain d'élection pour ces échanges de répliques ${ }^{34}$. Dans la nouvelle 32 , au bon mot s'ajoute une métaphore dans la vengeance d'une épouse : après que son mari s'est excusé de n'avoir pu remplir son devoir conjugal parce qu'il «avoit couru la poste»,

\footnotetext{
${ }^{28} N R 6,9,18,19,21,31,32,60,62,64$ et 90 .

${ }^{29}$ NR $6,9,60$ et 90 .

${ }^{30}$ Rhétorique, II, 2, 1378 I, trad. de C. E. Ruelle revue par P. Vanhemelryck, Livre de Poche, 1991, p.184.

${ }^{31}$ Balthazar Castiglione, Livre du Courtisan, trad. G. Chappuis, revue par Alain Pons, G.-F., 1991, II, 60, p. 181-182.

${ }^{32} N R$, p. 105.

${ }^{33} N R$, p. 147.

${ }^{34}$ Quelques nouvelles sont constituées par un échange de répliques, telles $N R 58,65$, et 63, qui présente un échange entre une harengère et un régent, associés par un chiasme sonore.
} 
et que le lendemain, il se moque d'un coq prenant le temps de «mugueter» une poule, « la dame luy respond, 'eh monsieur pardonnez luy, peult estre qu'il ha couru la poste toute la nuict' ${ }^{35}$. De telles vengeances ne suscitent aucune réprobation de la part du conteur puisque, tout en témoignant d'une conscience aiguë des pouvoirs et des mécanismes du langage, elles suscitent le plaisir du lecteur sans nuire à quiconque.

Le reste du temps, ce qui constitue le récit de vengeance est une beffa, c'està-dire une farce fondée sur une machination complexe, qui témoigne de l'ingéniosité et de l'intelligence du dupeur et aboutit à la déconfiture d'une dupe, souvent prédestinée, qui en subit dérision et dommage : le mari trompé devient le dupeur, et sa vengeance consiste à retourner l'injure subie. C'est ce qui se passe dans la nouvelle 60. Un maréchal trompé se venge du prêtre Jehan Melaine qui prend sa place au lit. Sa vengeance consiste à retourner contre le prêtre la tromperie subie, à lui faire croire qu'il est à sa forge (où il a en réalité envoyé sa femme), et à l'attendre dans la couche conjugale : «Incontinent que messire Jehan entendit battre à deux, il ne fut pas endormy: il se leva avec sa grosse robe de nuict, et entre par l'huys accoustumé, et se vient coucher aupres de ce mareschal, pensant estre aupres de sa femme $»^{36}$. Le prêtre se fait finalement étriller « à beaux nerfz de beuf ». De façon plus originale, la nouvelle 19 met en scène le savetier Blondeau, tourmenté par le singe de son voisin qui, imitant les gestes de l'artisan, vient chez lui lacérer son cuir, et le prive ainsi de son gagne-pain. Après avoir bien observé le singe, Blondeau lui donne à imiter un geste destiné à le blesser ${ }^{37}$. Le singe, qui «vouloit s'esbatre à ce nouveau passetemps qu'il n'avoit point encore veu faire», est ainsi amené à s'égorger en passant plusieurs fois la lame du rasoir près de son cou. Parce qu'il confère à sa nouvelle la structure d'une expérimentation ${ }^{38}$ (observation, déduction, expérience), le conteur suggère qu'il s'intéresse moins à la passion vindicative comme telle qu'aux ruses qu'elle conduit à déployer. On aurait tort d'en déduire pour autant qu'il plaide pour la passion vindicative.

\section{Les prises de parti du narrateur}

Alors que la vengeance constitue le terreau le plus fertile des Histoires tragiques - en particulier celles de Pierre Boaistuau, parues en 1559-qui sont presque contemporaines des Nouvelles Récréations, les contes relatant une vengeance sont traités avec une certaine légèreté par Bonaventure des Périers, qui met en application les remèdes préconisés contre les chagrins dans le prologue de son recueil. L'épitaphe du savetier Blondeau, qui conclut la nouvelle $19^{39}$, ne souffle mot de la vengeance assouvie contre le singe alors qu'elle évoque le premier récit relatif aux pièces d'argent trouvées par le savetier. De même, la dernière nouvelle du recueil, qui relate l'homicide prémédité d'un mari trompé, fait entendre le

\footnotetext{
${ }^{35} N R$, p. 149.

${ }^{36} N R$ 60, p. 224.

${ }^{37} N R$, p. 98 : «Pour le faire adviser au singe ».

${ }^{38}$ Voir Guy Demerson, «Bonaventure des Périers : la nouvelle, art de patience», Prose et prosateurs de la Renaissance, Mélanges offerts à Robert Aulotte, Paris, SEDES, 1988, p. 72. ${ }^{39} \mathrm{NR}, \mathrm{p} .99$.
} 
commentaire d'un conteur qui se tient résolument à distance des faits : «Mais que voulez-vous ? Il fasche à un mary d'estre cocu en sa propre personne $»^{40}$.

Cette légèreté ne doit cependant pas nous abuser: pour discrètes qu'elles soient, les prises de parti du conteur sont indéniables. Il arrive ainsi que, sans la moindre ambiguïté, il souligne combien la vengeance est abusive. La nouvelle 56 met aux prises un gentilhomme coupant l'oreille à un voleur qui lui a dérobé les boutons de son habit et qui réplique au coupe-bourse : «Rendz moi mes boutons, je la te rendray». Elle est conclue par une intervention du narrateur qui commente ainsi le geste du gentilhomme: «Il ne luy faisoit pas mauvais party, s'il eust pu recoudre son oreille, comme le gentilhomme ses boutons». Il souligne ainsi la disproportion d'une vengeance apparentée à la loi du talion qui établit une fausse équivalence entre deux choses sans commune mesure. Thomas d'Aquin soulignait la part de raison et de justice qui entre dans la vengeance quand elle comporte une relation entre la peine qu'on veut infliger et le dommage subi ${ }^{41}$. Mais dès lors que la vengeance est sans commune mesure avec l'offense qu'elle prétend châtier, l'acte vindicatif mérite de s'appeler plutôt cruautét ${ }^{4}$. C'est cette disproportion que souligne Des Périers quand il relate la vengeance du prévôt La Voulte dans la nouvelle 80. Alors même qu'il vient de saisir un coupe-bourse, il se fait voler par un autre déguisé en gentilhomme, qu'il ne peut donc identifier. Faute de pouvoir retrouver son voleur, «par un beau depit, en fit pendre une douzaine d'aultres qu'il tenoit prisonniers: et puis leur fit faire leur proces $»^{43}$. Propulsée en tête de phrase, l'explication psychologique discrédite l'exécution décidée de façon arbitraire et inique. Douze personnes paient pour une, et le châtiment précède l'instruction. On hésite d'ailleurs à parler de châtiment puisque, si l'on en croit Aristote, «Dans le châtiment, on considère celui qui le subit, tandis que dans la vengeance, on a plutôt souci de celui qui l'exerce, le but de celui-ci étant de se donner une satisfaction $»^{4}$.

De façon plus générale, le narrateur qui recommandait dans son préambule de ne pas se fâcher d'une chose irrémédiable rappelle qu'aucun plaisir acquis par une vengeance ne saurait contrebalancer la vie : «c'est un povre reconfort à un homme, que sa mort en sera bien vengée $\gg^{45}$. Il commente ainsi la bêtise d'un bâtard refusant le secours d'un témoin à seule fin de se venger d'un prévôt qui commet une erreur judiciaire : «Pensez le grand sens d'où il estoit plein, de se laisser pendre, Et qu'il en eust esté bien vengé ${ }^{46}$. La nouvelle 41 , qui précède immédiatement celle-ci, rappelait d'ailleurs que la colère est une humeur, au même titre que la mélancolie, et

${ }^{40} N R$, p. 312.

${ }^{41}$ Thomas d'Aquin, ouvrage cité, $\mathrm{I}^{\mathrm{a}} \mathrm{II}^{\mathfrak{x}}$, quest. 46 , art. 4.

${ }^{42} \mathrm{Un}$ vol de plus de cinq sous méritait la mort, au $\mathrm{XVI}^{\mathrm{e}}$ siècle, et beaucoup soulignaient l'iniquité de cette peine. Voir A. Laingui et A. Lebigre, Histoire du droit pénal, Paris, Cujas, 1979, p. 126.

${ }^{43} N R$, p. 285.

${ }^{44}$ Rhétorique, I, 10, 1369b 17, éd. citée, p. 143.

${ }^{45} N R 44$, p. 185.

${ }^{46} N R$ 44, p. 184. 
dont le conteur cherche précisément à purger ceux auxquels s'adressent ses Joyeux propos $^{47}$.

La vengeance n'est légitime que lorsque son accomplissement chasse un chagrin : c'est le cas de la nouvelle $18^{48}$, dans laquelle le menuisier Gillet se venge du lévrier de son riche voisin, qui vient régulièrement lui dérober son dîner. La présentation du personnage de Gillet par le narrateur ne ménage pas les marques de sympathie : l'artisan, auquel manque le secours d'une épouse puisqu'il est veuf, et qui est père d'une fillette, «[...] travailloit pour gaigner sa vie le mieulx qu'il pouvoit ». En même temps que son courage, est soulignée son indépendance : «Et se trouvoit aussi bien de son petit ordinaire comme un plus riche du sien $»^{49}$, et le narrateur prend parti contre son voisin, le propriétaire du lévrier ${ }^{50}$. Dans l'impossibilité de se plaindre des méfaits du lévrier, Gillet «proposa de s'en venger $»^{51}$. Il le rosse d'importance, un jour en secret Le narrateur nous fait connaître la disparition du lévrier dans une phrase conclusive: « [...] il avoit accoustré tellement le levrier, que le gentilhomme ne mangea depuis lievre de sa prise $\aleph^{52}$. En évoquant les privations culinaires que subit le riche voisin, le narrateur prend parti pour Gillet, dont précisément les repas étaient, avant la vengeance, dévorés par le chien. Pourvu qu'ils le fassent avec astuce, il est permis aux humbles de prendre une revanche $^{53}$. De même, le savetier Blondeau ne se venge du singe qui lui gâte son travail que pour chasser cette mélancolie qui ne l'a atteint que deux fois, lorsqu'il s'est trouvé en possession de pièces d'argent en abondance, et quand le singe de son voisin est venu lui chercher noise : "Quand il en fut bien ennuyé, il delibera de s'en venger $\aleph^{54}$. En prenant parti pour Blondeau, le narrateur semble se rallier à l'avis d'Aristote selon lequel le véritable auteur du dommage est celui qui provoque la colère $^{55}$. On peut même se demander si la vengeance de ce personnage mérite d'être qualifiée de passion colérique: Blondeau en effet ne se précipite pas, il calcule, mesure, et alors seulement agit. Or, dans son traité De la Colère, Sénèque fait de la colère le propre de ceux qui ne maîtrisent pas les événements ${ }^{56}$.

\footnotetext{
${ }^{47} N R$, p. 174: «Il y ha une maniere de gens qui ont des humeurs colericques, ou melancolicques, ou flegmathicques...».

${ }^{48} N R$, p. $94-96$.

${ }^{49} N R$, p. 94.

${ }^{50} N R$, p. 94 : « S'il est trop riche, il vous tiendra en subjection, et vous faudra endurer de luy ».

${ }^{51} N R$, p. 95.

${ }^{52} \mathrm{NR}, \mathrm{p} .96$, dernière phrase de la nouvelle.

${ }^{53}$ Krystyna Kasprzyk écrit ainsi «les situations où les gens du peuple remportent une victoire sur les représentants des classes supérieures sont assez fréquentes », Introduction, p. XXXIX.

${ }^{54} \mathrm{NR}, \mathrm{p} .98$.

${ }^{55}$ Aristote, Éthique à Nicomaque, V, vIII, 9, trad. J. Voilquin, G.-F., 1992, p. 156.

${ }^{56}$ Sénèque, De Ira, I, XI, 5, Entretiens, Lettres à Lucilius, éd. P. Veyne, Robert Laffont (coll. Bouquins), 1993, p. 117 (Quo alio Fabius adfectas imperii uires recreauit quam quod cunctari et trahere et morari sciit, qua omnia irati nesciunt ?), mais aussi II, XXIX, 1, p. 146 (Maximum remedium irce mora est. Hoc ab illa pete initio, non ut ignoscat sed ut iudicet: graues habet impetus primos; desinet, si expectat).
} 
À cet effet de purgation du chagrin, la vengeance doit ajouter l'inventivité pour intéresser Des Périers. Il affectionne particulièrement les vengeances fondées sur une imitation de l'offenseur par l'offensé, qui tend un filet de façon que le trompeur coure se tromper lui-même ${ }^{57}$. Le narrateur met en valeur l'astuce déployée, et ce qui occupe le devant de la scène est moins la furie vengeresse que l'intelligence déployée par la victime de l'offense. La nouvelle 90 met en scène un mari trompé soucieux de se venger de son épouse. Agissant comme le savetier Blondeau avec prévoyance et prudence, il prépare un guet-apens pour punir son épouse adultère. Mais il ne fait rien lui-même: il agit par délégation. Il s'adresse à son valet (première délégation) pour faire assoiffer la mule qui transportera sa femme, afin de l'amener à boire (seconde délégation) et à noyer sa femme. Ces deux délégations auxquelles recourt l'époux offensé confèrent à l'acte vindicatif un caractère très indirect, qui semble avoir retenu l'attention du narrateur : le récit était en effet déjà présent dans la nouvelle 36 de l'Heptaméron, mais Marguerite de Navarre insistait au contraire sur la responsabilité des hommes dans leurs actes ${ }^{58}$. Le récit de vengeance semble ainsi intéresser Des Périers à condition que la passion vindicative touche la victime visée par un biais, un détour ou encore par procuration : la tâche du narrateur consiste alors à faire sa place à l'inattendu, en réservant la surprise du procédé. Dans la nouvelle 19, Blondeau amène le singe à se trancher la gorge lui-même, tandis que l'épouse frustrée de la nouvelle 32 prend la défense du coq pour reprocher à son mari son impuissance.

Le caractère indirect et rusé de la vengeance ne suffit pourtant pas à la légitimer. La discrétion du narrateur à la fin de la nouvelle 90 ne doit pas faire oublier qu'il a annoncé son récit en présentant, parmi les divers types de cocus, ceux qui « le sçavent et s'en vengent» comme «mauvais folz et dangereux $»^{59}$. Il semble que Des Périers estime plus légitime et plus plaisante la vengeance qui se contente de répondre à l'offense avec grâce et sans violence, dont la nouvelle 64 fournit une plus longue illustration. Cette nouvelle (p. 237-244) rapporte l'histoire d'un jeune homme amoureux d'une coquette qui ne parvient pas à lui faire rendre les armes, bien qu'il passe par toutes les conditions qu'elle lui impose. Il se décide à feindre la folie et il devient son amant. Dans un tercet rimé, elle ridiculise en public son amoureux transi :

Que diriez-vous d'un verd vestu,

Qui a baisé sa femme au cu,

En luy faisant hommage. ${ }^{60}$

À quoi le fol, qui avait pris le parti de répéter toujours « Ha ha formage » se venge à la fois de l'épigramme et de la femme en répliquant:

Que diriez-vous d'un verd vestu,

\footnotetext{
${ }^{57}$ Voir B. Castiglione, Livre du Courtisan, éd. citée, II, 85, p. 208.

${ }^{58}$ «Et les hommes et les femmes sont communs aux vices et aux vertus », Heptaméron, éd. M. François, Garnier frères, 1967, p. 263.

${ }^{59} N R$, p. 310.

${ }^{60} N R$, p. 242.
} 
Qui a damé sur vostre cu

Disant : «Ha ha formage ? ${ }^{61}$

Et le narrateur de commenter: «le jeune homme se trouva vengé d'elle à un bon coup : de toutes les cautelles du temps passé ».

Dans cette nouvelle, la vengeance, purement verbale, a l'approbation du narrateur qui dénonçait les coquetteries de la femme puisqu'il ne les expliquait pas par une rigueur morale: «Car les rusées ont celle façon de tenir tousjours quelqu'un des poursuyvantz en langueur, pour faire couverture à la jouissance qu'elles donnent aux aultres $»^{62}$. L'ingéniosité $d u \ll$ fol » lui a permis de vaincre une résistance sans loyauté, puis de prendre une revanche. Son astuce se trouve d'autant plus justifiée que l'attaque à laquelle il répond a été préméditée ${ }^{63}$, tandis que sa réplique est présentée à maintes reprises comme venue des dieux : «tout remply d'une fureur poeticque », il « vint respondre promptement à la dame ». En conclusion de son récit, Des Périers souligne la victoire de l'inspiration poétique sur le froid calcul de la coquette: "Car les dieux envoyent leur ayde et faveur aux amoureulx qui ont bon cueur, comme il se peult veoir de ce jeune homme: auquel Phebus donna l'esprit poeticque pour respondre promptement, en se deffendant contre le blason que sa dame avoit si finement et deliberément songé contre luy ${ }^{64}$. À la préméditation de la vengeance, nourrie par la rancune (celle de la dame), le narrateur oppose l'esprit et le «bon cueur», qui se caractérisent par une liberté garante de la hauteur de vues.

Mais si le narrateur reproche à la femme moqueuse d'avoir fait «trop les difficiles, et les asseurées », d'avoir infligé à son prétendant une humiliation intime (puisqu'elle se fait «baiser le cul »), il la blâme surtout, semble-t-il, d'avoir ajouté à l'offense privée l'injure publique, puisqu'elle a attaqué le jeune homme en société. Or la vengeance ne tire pas à grave conséquence quand elle s'en tient à une relation privée. Il en va tout autrement quand elle engage l'honneur des individus dans la sphère sociale.

\section{Vengeance et patience}

À la lecture de cette nouvelle 64, il est permis de se demander si Des Périers n'incline pas, en réalité, à condamner la vengeance tant que l'offense n'est pas publique. Cette déduction nous paraît confirmée par la nouvelle 9. Nous y est relaté l'adultère que commet une sotte séduite par un certain sire André qui lui promet de «terminer l'oreille de son enfant». Quand son mari revient et qu'il apprend son infortune, il passe la nuit dans des vengeances imaginaires et donne «par fantasie plus de cent coups de dague à l'acheveur ${ }^{65}$. Confronté à son « rival », il lui dit mille outrages, puis se trouve « contraint de s'appaiser par une couverte de Cataloigne que luy donna sire André ». Cet évitement de la vengeance s'explique par plusieurs raisons. Tout d'abord, le mal étant irrémédiable, le chagrin apparaît comme inutile.

${ }^{61} N R$, p. 243.

${ }^{62} N R$, p. 239.

${ }^{63} N R$, p. 242: «Elle vint dire d'une grace affaitée ce qu'elle avoit premedité des le paravant $»$.

${ }^{64} N R$, p. $243-244$.

${ }^{65} \mathrm{NR}, \mathrm{p} .55$. 
Par ailleurs, l'offense a un caractère privé et aucune rumeur ne l'a colportée. Enfin, l'adultère commis avec sire André n'entraîne pas les conséquences successorales ordinairement redoutées: la femme étant déjà enceinte ne court pas le risque d'enfanter un bâtard qui hériterait du mari. L'arrière-plan philosophique de cette nouvelle paraît influencé par l'analyse sénéquienne de l'outrage, qui ne doit pas être considéré comme plus qu'une blessure d'amour-propre ${ }^{66}$, si bien qu'une réparation financière suffit à le compenser. C'était également l'idée de Castiglione, protestant que l'adultère n'est pas une faute si grave ${ }^{67}$. D'autant plus que pendant la veille nocturne, une scène de colère in abstentia a permis de désamorcer la passion vindicative $^{68}$

Inversement, le mari trompé de la nouvelle 90 commet un acte de vengeance privé appelé en droit «homicide sur fornication». Des Périers, qui a repris la quarante-septième des Cent Nouvelles et la nouvelle 36 de l'Heptaméron, a supprimé le caractère public de l'affront (nul n'est informé de l'inconduite de l'épouse), ce qui accentue la cruauté de l'homicide. Il a encore pris soin de priver son protagoniste de toute circonstance atténuante de façon à faire apparaître sa vengeance non comme un châtiment, mais comme un obstacle à récidive. Cette ultime nouvelle signifie donc une condamnation du personnage vindicatif ${ }^{69}$, à la fois rancunier parce qu'il se trouve dans une colère permanente «à cause de la persistance de la tristesse qu'il tient enfermée en lui-même $»^{70}$, et implacable parce que seule la punition peut apaiser son violent désir de vengeance.

Faut-il en déduire que Des Périers encourage le recours à la patience ? Nombre de ses nouvelles proposent une réflexion sur la patience, comme celle qui ouvre la nouvelle 69 dans laquelle un rustique poitevin sait différer la réponse à des questions sur un itinéraire ${ }^{71}$. Lisant dans les nouvelles de Bonaventure des Périers une opposition au traité De Cohibenda ira de Plutarque, Robert Aulotte définit la patience comme l'attitude par laquelle le passionné manifeste la maîtrise de soi $^{72}$, alors que Lionello Sozzi ${ }^{73}$ voit dans les Nouvelles Récréations une exhortation à la

\footnotetext{
${ }^{66}$ Sénèque, De Constantia, V, 1 et 2, éd. citée, p. 318-319 (Diuidamus, si tibi uidetur, [...] iniuriam a contumelia, et Ad tantas ineptias peruentum est ut non dolore tantum sed doloris opinione uexemur).

${ }^{67}$ Livre du Courtisan, III, 50, éd. citée, p. 290.

${ }^{68}$ Sénèque souligne déjà l'utilité qu'il y a à feindre la colère, De Ira, II, XVII, 1, éd. citée, p. 138 (ubicumque alieni animi ad nostrum arbitrium agendi sunt, modo iram, modo metum, modo misericordiam, ut aliis incutiamus, ipsi simulabimus, et sape id quod ueri adfectus non effecissent effecit imitatio adfectuum).

${ }^{69}$ Condamnation qui rappelle bien sûr celle de Picrochole par Rabelais.

${ }^{70}$ Thomas d'Aquin, ouvrage cité, $\mathrm{II}^{\mathrm{a}} \mathrm{II}^{\mathfrak{x}}$, quest. 128, art. 5, sol. 2.

${ }^{71} N R$, p. 253 : «Il y ha beaucoup de manieres de s'exercer à la patience ».

${ }^{72}$ Robert Aulotte, «Fous et farceurs dans les Nouvelles Récréations de Bonaventure des Périers ", Le Génie de la forme, Mélanges de langue et littérature offerts à Jean Mourot, Presses universitaires de Nancy, 1982, p. 97-106 : «La domination chèrement acquise sur ses passions conduit l'homme à se consumer dans un feu intérieur qu'il n'ose pas manifester; et l'absolue taciturnité l'emprisonne dans des idées vénéneuses », p. 97.

${ }^{73}$ Lionello Sozzi, Les Contes de Bonaventure des Périers: Contribution à l'étude de la nouvelle française de la Renaissance, Turin, 1965, p. 401.
} 
patience comme vertu intellectuelle confiée à l'auto-contrôle et au culte de l'intériorité. Cherchant à vérifier cette hypothèse, Guy Demerson, qui distingue les formes de patience ${ }^{74}$, s'interroge sur la signification profonde de cette patience : estelle refoulement dangereux, répression de l'élan vital, ou vertu des âmes désenchantées qui recherchent la paix intérieure ? Il oublie cette forme de la patience proche de la vertu, que saint Augustin opposait à la vengeance ${ }^{75}$, et saint Thomas à la tristesse, passion qui s'éveille au contact d'un mal présent ${ }^{76}$. Paradoxalement, alors que le genre de la nouvelle donne une scène à la représentation de la ruse, il n'est pas si éloigné qu'on pourrait le croire de l'attitude de la patience. On se souvient ainsi que c'est à Boccace que fut décerné le titre de «docteur de patience en adversité», parce que la patience de Griselda dans la dernière nouvelle du Décaméron rappelait celle de Job à qui tout a été donné puis ôté. La patience à laquelle encouragent certaines des Nouvelles Récréations ressemble à la charité ${ }^{77}$ qui «souffre tout et est patiente et courageuse ». Elle «sait regarder de haut les choses capables de l'affecter de chagrin $\gg^{78}$, et n'a rien à voir avec la patience du mari comploteur de la dernière nouvelle ${ }^{79}$.

C'est cette patience aux antipodes de la vengeance qui anime le mari trompé de la nouvelle 6. Devant la certitude de l'infidélité de sa femme, il «s'armoit de patience», considérant la folie qu'il a faite d'épouser une toute jeune fille ${ }^{80}$. Pourtant, l'adultère de son épouse est connu de beaucoup, ce qui pourrait légitimer une vengeance qui, répondant à une offense publique, serait défense de l'honneur. La famille du mari vient lui réclamer d'en prendre réparation, et oppose la rumeur à sa patience : «s'il n'y obvioit: il donneroit à penser qu'il seroit de vil courage $»^{81}$. Deux des Cent Nouvelles nouvelles ont pu inspirer à Des Périers ce sujet: la nouvelle 49, présentant la vengeance d'un marchand d'Arras qui, bien informé de l'inconduite de sa femme, la déshonore publiquement, et la nouvelle 71 dans laquelle un mari, s'avisant de son infortune, demande à son épouse d'agir avec plus

\footnotetext{
${ }^{74}$ La persévérance du savoir, la tolérance $(N R$ 4), la constance du prudent $(N R$ 6), et la stratégie lucide contre l'impatience, qu'il voit précisément à l'œuvre dans $N R 90$, art. cité, p. 66-67.

${ }^{75}$ Augustin, De Patientia, chapitre 9 en particulier.

${ }^{76}$ Thomas d'Aquin, ouvrage cité, II $^{\mathrm{a}} \mathrm{II}^{\mathfrak{x}}$, quest. 136.

77 Voir mon article «Patience ou passion dans la dernière des Nouvelles Récréations de Bonaventure des Périers », Réforme, Humanisme, Renaissance, déc. 2008.

${ }^{78}$ Cicéron, Tusculanes, III, VII, 14 et 15, éd. citée, p. 299 (Non cadunt autem haec in virum fortem: igitur ne agritudo quidem. At nemo sapiens nisi fortis: non cadet ergo in sapientem agritudo. Praterea necesse est, qui fortis sit, eundem esse magni animi; qui autem magni animi sit, invictum; qui invictus sit, cum res humanas despicere atque infra se positas arbitrari).

${ }^{79}$ Que saint Augustin appelle la patience du méchant, telle celle de Catilina, De Patientia, chapitres 3 et 5 .

${ }^{80} N R$, p. 40 : «Songeant en luymesme qu'il falloit porter la penitence de la follie qu'il avoit faicte». Dans $N R 16$, p. 82, un autre personnage déclare qu' «il ne faut pas se garder tant qu'on soit usé, pour prendre femme. Car il n'est rien qui ouvre la porte plus grande à cocuage, que l'impuissance du mary ».

${ }^{81} N R$, p. 40.
} 
de discrétion. Mais aucune de ces nouvelles ne proposait de réflexion morale, de retour du mari sur lui-même, ou encore de critique de la rumeur. Dans la nouvelle 6 de Des Périers, l'opinion publique produit un double effet : c'est elle qui fait sentir le chagrin $^{82}$ et le produit dans l'âme, c'est elle encore qui discrédite la patience ou la passivité du mari trompé. Le narrateur s'applique d'ailleurs à souligner la distorsion entre la sphère publique - ou domaine de l'opinion - et le for intérieur. L'analyse de l'offense semble combiner l'explication stoïcienne et l'interprétation chrétienne. Sénèque établissait une distinction entre l'injure de l'agresseur (iniuria, véritable outrage), et l'insulte (contumelia, blessure d'amour-propre) qui tient à l'idée que s'en fait la victime ${ }^{83}$. Ainsi, la colère ne naît pas de l'offense mais de l'opinion qu'on nous a offensés ${ }^{84}$, et le désir de vengeance vient de la médiocrité d'une âme qui se sent diminuée par une action ou une parole qui la déconsidère ${ }^{85}$. L'offense ne dépend pas de l'offenseur, et c'est pour des raisons internes (respect de la vertu, conscience morale) que la faute doit être évitée. Le mari trompé se refuse à châtier sa femme parce qu' «il pensoit que la femme se deust garder par un respect de la vertu, et par crainte de son deshonneur ${ }^{86}$ : autrement toutes les murailles de ce monde ne la sçauroyent tenir qu'elle ne fist une fois des siennes», et «il raisonnoit en soymesmes que l'honneur d'un homme tiendroit à bien peu de chose, s'il dependoit du fait d'une femme ${ }^{87}$.

Par cette nouvelle, qui met en scène la patience d'un époux ${ }^{88}$, Des Périers semble apporter un écho à la nouvelle 37 de Marguerite de Navarre. On peut en effet imaginer, comme le fait Robert Muchembled ${ }^{89}$, que Marguerite de Navarre et Bonaventure Des Périers se lisaient mutuellement les récits qu'ils écrivaient. Cette nouvelle 37 succédait immédiatement et significativement à la nouvelle 36 , source de la dernière des Nouvelles Récréations qui relate la vengeance impitoyable et froide d'un mari trompé. Comme pour répondre à cette brutalité, la nouvelle 37

\footnotetext{
${ }^{82}$ Voir Cicéron, Tusculanes, III, XI, 24, éd. citée, p. 303 : «la cause qui produit le chagrin dans l'âme est l'opinion» (Est igitur causa omnis in opinione).

${ }^{83}$ Sénèque, De Constantia, V, 2, éd. citée, p. 318-319 : ce n'est pas seulement la souffrance qui nous ronge, mais l'idée de la souffrance (Ad tantas ineptias peruentum est ut non dolore tantum sed doloris opinione uexemur).

${ }^{84}$ Sénèque, De Ira, I, III, 1, éd. citée, p. 111 ('Irascimur' inquit 'sape non illis qui laserunt, sed iis qui lasuri sunt; ut scias iram non ex iniuria nasci.' Verum est irasci nos lasuris, sed ipsa cogitatione nos ladunt, et iniuriam qui facturus est iam facit), et II, XXII, 2, p. 141 (causa autem iracundia opinio iniuria est, cui non facile credendum est).

${ }^{85}$ Sénèque, De Constantia, X, 3, éd. citée, p. 325 : «On montre bien peu d'intelligence, et en même temps bien peu de fierté, quand on s'affecte d'une offense » (Itaque nec prudentice quicquam in se esse nec fiducia ostendit qui contumelia adficitur; non dubie enim contemptum se iudicat).

${ }^{86}$ C'est là le raisonnement de B. Castiglione dans le Livre du Courtisan, III, 41, éd. citée, p. 277: «Le grand frein qui retient les femmes est l'amour de la vertu et le désir de l'honneur».

${ }^{87} N R$, p. 41.

${ }^{88}$ Dont le roi, auditeur du récit, juge qu'il est «l'un des plus froidz et plus patiens hommes », NR, p. 43.

${ }^{89}$ Robert Muchembled, L'Invention de l'homme moderne, Paris, Fayard, 1988, p. 90.
} 
présente Mme de Loué, épouse trompée qui, au lieu de se venger de son mari, choisit de lui donner ses aises chez sa maîtresse. De Thou résume la nouvelle ainsi : «Mme de Loué, par sa grand'patience et sa longue attente, gagna si bien son mari qu'elle le retira de mauvaise vie». Le récit est suivi par le commentaire des devisants et Parlamente s'indigne: "J'ai opinion qu'en mariage [patience] amène enfin inimitié, parce qu'en souffrant injure de son semblable on est contraint de s'en séparer le plus qu'on peut, et de cette étrangeté-là vient un dépris de la faute du déloyal, et en ce dépris peu à peu l'amour diminue $»^{90}$. Autrement dit, Parlamente condamne la patience de l'épouse au nom de l'amour conjugal et de l'honneur qui doit lui rester attaché, alors que Des Périers paraît justifier la patience de son mari trompé par des arguments stoïciens sur la nature de l'injure. À cela s'ajoute en filigrane une distinction entre ce que requièrent le regard d'autrui (les proches de l'époux trompé réclament la vengeance), et le regard intérieur par lequel le mari mesure sa responsabilité dans son malheur. Là où Marguerite de Navarre mettait l'accent sur l'amour conjugal, Des Périers refuserait ce qu'a d'aliénant le regard des autres $^{91}$.

Il en tient cependant compte, et nous allons voir comment en analysant la signification du remède que l'époux propose à sa femme. Celui-ci engage son épouse à le tromper discrètement, en introduisant ses amants par un huis à l'arrière de sa maison. On hésite à voir dans cette attitude de dissimulation un respect de la rumeur; traduit-elle un refus de l'amplification que donne à une faute son exhibition $^{92}$ ? La discrétion que le protagoniste demande à sa femme l'amène à s'amender et à «prendre vergoigne de son faict $»^{93}$. En lui proposant de dissimuler ses tromperies, l'époux lui suggère et lui inspire simultanément la honte devant son crime $^{94}$

Il y a là un refus du scandale ${ }^{95}$ que l'on retrouve dans la nouvelle 62 , qui ne traite pas de vengeance, mais affronte à la supérieure d'un couvent un jeune homme déguisé en sœur Toinette. Lorsque la vérité éclate, «il fallut y remedier par patience, car elle n'eust pas voulu scandalizer la religion $»^{96}$. Le scandale est en effet péché contre la charité et la correction fraternelle, puisqu'en provoquant le prochain, il le

\footnotetext{
${ }^{90}$ Marguerite de Navarre, Heptaméron, éd. citée, p. 263.

${ }^{91}$ Daniel Ménager propose une tout autre analyse de cette nouvelle 6, dans Les Nouvelles Récréations et Joyeux Devis de Bonaventure Des Périers, «La nouvelle 6 », Université de Clermont-Ferrand, sous la direction de Dominique Bertrand et Bénédicte Boudou, automne 2008.

${ }^{92}$ Le droit du $\mathrm{XVI}^{\mathrm{e}}$ siècle jugeait que la dissimulation atténuait la gravité du délit, contrairement au droit ancien qui juge moins grave un acte accompli ouvertement. Voir A. Tiraqueau, De Pœnis legum ac consuetudinum, statutorumque temperandis, aut etiam remittendis, et id quibus quotque ex causis, Lyon, C. Senneton, 1559, Paris, Economica, éd. A. Laingui, 1986, Cause 57, 4. Voir aussi sur ce point l'analyse de Daniel Ménager, art. cité. ${ }^{93} \mathrm{NR}, \mathrm{p} .42$.

${ }^{94}$ Thomas d'Aquin, ouvrage cité, $\mathrm{II}^{\mathrm{a}} \mathrm{II}^{\mathfrak{x}}$, quest. 66, art. 3 : le caractère occulte du péché le diminue parce que d'une part il est la marque de la honte, d'autre part parce qu'il supprime le scandale. On peut penser à la parole de saint Paul (I, Cor., 7, 14) : le mari sanctifie sa femme.

${ }^{95}$ Matth. 18, 7 (et Luc, 17, 1) «Malheur à l'homme par qui le scandale arrive ».

${ }^{96} N R$, p. 232.
} 
fait entrer dans le mal ${ }^{97}$. La supérieure du couvent empêche donc que l'erreur et la supercherie, reconnues dans la sphère privée, ne soient découvertes au grand jour où elles donneraient un mauvais exemple. Le mari patient de la nouvelle 6 cherchait à faire passer dans la sphère privée l'offense publique de son épouse, qui aurait légitimé des intentions vindicatives. Ainsi donc, non seulement cet époux substitue à la vengeance la patience et la mansuétude, non seulement il privilégie le retour sur soi et la conscience de sa propre faute, mais il prend en compte les répercussions que ses actes et ceux de son épouse peuvent avoir sur ceux qui les entourent ${ }^{98}$.

Le prologue des Nouvelles Récréations et Joyeux Devis annonce la volonté récréative du conteur qui cherche à endiguer par le rire les chagrins des hommes. Parmi ces maladies de l'âme, son sonnet liminaire ${ }^{99}$ retient particulièrement la colère. Il la présente comme une passion sociale, et il met en situation les réactions vindicatives qu'elle entraîne. Tant que la nouvelle ressemble à une facétie parce que la vengeance tient en un jeu de mots astucieux, elle est admise par le conteur car elle montre une maîtrise de soi et un contrôle ordinairement incompatibles avec l'ire vengeresse qui déforme les traits du visage et suscite des propos violents. Il se méfie en revanche de la passion vindicative qui entraîne à la démesure et cherche plus à faire souffrir l'offenseur qu'à corriger les vices ${ }^{100}$. Mais là où les auteurs d'histoires tragiques prennent ouvertement et parfois lourdement parti pour ou contre la vengeance, Bonaventure des Périers s'abstient de rappeler que la vengeance, présente dans l'Ancien Testament ${ }^{101}$, est bannie du Nouveau. Il ne rappelle pas non plus la phrase de saint Paul réservant la vengeance à $\mathrm{Dieu}^{102}$. Il se contente de proposer deux fois plus d'offenses privées que d'offenses publiques, afin de suggérer que la vengeance n'offre de sens que devant un public ${ }^{103}$. Et lorsqu'il

\footnotetext{
${ }^{97}$ Thomas d'Aquin, ouvrage cité, $\mathrm{II}^{\mathrm{a}} \mathrm{II}^{\mathfrak{x}}$, quest. 43.

${ }^{98}$ Daniel Ménager, dont l'article est mentionné dans la note 92, souligne de son côté que le roi, dans ce récit encadré (le seul des $N R$ ), salue la dissimulation du personnage et le promeut.

${ }^{99} N R$, p. 2 : «Laissez à part vostre chagrin, vostre ire ».

${ }^{100}$ Thomas d'Aquin, ouvrage cité, $\mathrm{II}^{\mathrm{a}} \mathrm{II}^{\mathfrak{x}}$, quest. 158, art. 1, sol. 3 .

${ }^{101}$ Sauf dans les livres de Job, du Siracide et du Qohéleth.

${ }^{102}$ Romains, 12, 19.

${ }^{103}$ Éric Méchoulan, «La dette et la loi : considérations sur la vengeance », Littératures classiques, $\mathrm{n}^{\circ}$ 40, 2000, p. 280.
} 
conclut son recueil par une vengeance homicide qui a les apparences de la justice (nouvelle 90), il évite de porter une condamnation directe, et préfère se demander si l'adultère est vraiment un tort ${ }^{104}$. La distance qu'il conseille aux lecteurs de prendre par rapport à leurs malheurs n'annonce pas la fuite dans le monde que dénoncera Pascal en fustigeant le divertissement, elle ressemble au détachement du cynique, ou à cette «invincible constance » qui est le dernier mot de son recueil ${ }^{105}$.

Bénédicte Boudou Université de Picardie Jules Verne

${ }^{104} N R$, p. 312.

${ }^{105} N R$, p. 313 : sonnet final. 